\title{
Gestión estratégica de microempresas del sector agroindustrial en Tabasco, México*
}

\section{Susana Suárez Reyes, María del Carmen Sandoval Caraveo, Clara Luz Lamoyi Bocanegra}

\author{
Universidad Juárez Autónoma de Tabasco - México \\ División Académica de Ingeniería y Arquitectura, Universidad Juárez Autónoma de Tabasco - México \\ División Académica de Ciencias Económico Administrativas, Universidad Juárez Autónoma de Tabasco - México
}

Las microempresas agroindustriales representan uno de los sectores fundamentales en la economía de México: generan empleos, y contribuyen al desarrollo regional y nacional. Sin embargo, las limitaciones y el corto tiempo de vida de estas empresas son preocupantes. El estudio presenta un análisis de la gestión estratégica de 194 microempresas del sector agroindustrial en Villahermosa, Tabasco, México. Los resultados muestran que estas empresas presentan deficiencias en dos de las etapas de dicho proceso: la formulación y la implementación de estrategias. Se ha identificado que los gerentes no planifican sus actividades, ni establecen sus objetivos a corto, mediano y largo plazo. Además, se ha detectado la falta de una filosofía organizacional, lo que puede afectar el funcionamiento óptimo de la organización.

Palabras clave: microempresas, manufacturas, agroindustrias, gestión estratégica

\section{Strategic management of microenterprises in the agroindustrial sector of Tabasco, México}

Agroindustrial microenterprises are one of the key sectors in the country's economy, generating jobs and contributing to regional and national development, however, the limitations and short lifespan these companies have is worrying. The study presents an analysis of the strategic management of 194 microenterprises in the agro-industrial sector of Villahermosa, Tabasco. The results show that these companies have deficiencies at two stages of this process, the formulation and implementation of strategies, as managers do not plan their activities or set their short, medium and long goals. The lack of an organizational philosophy is detected, which can affect the optimal functioning of the organization.

Keywords: microenterprise, manufactures, agroindustries, strategic management

\section{Gestão estratégica de microempresas do setor agroindustrial de Tabasco, México}

As microempresas agroindustriais representam um dos setores fundamentais da economia do país, pois geram empregos e contribuem para o desenvolvimento regional e nacional, porém, as limitaçóes e a curta vida útil dessas empresas são preocupantes. O estudo apresenta uma análise da gestão estratégica de 194 microempresas do setor agroindustrial em Villahermosa, Tabasco, México. Os resultados mostram que essas empresas apresentam deficiências em duas das etapas

* Este artículo reporta los resultados obtenidos en la tesis Estrategias gerenciales de las micro y pequeñas empresas agroindustriales de Villahermosa, Tabasco presentada por Susana Suárez Reyes para obtener el grado de Maestra en Administración y Dirección Estratégica. 
do referido processo, a formulação e implementação de estratégias, identificando-se que os gestores não planejam suas atividades ou estabelecem seus objetivos a curto, médio e longo prazo. É detectada a falta de uma filosofia organizacional, o que pode afetar o funcionamento ideal da organização.

Palavras-chave: microempresas, fabrica, agroindústrias, gestão estratégica

\section{Introducción}

En la actualidad, las microempresas se han convertido en unidades económicas de gran importancia para la economía nacional e internacional. De acuerdo con el Instituto Nacional de Estadística y Geografía (INEGI, 2014), en México, las microempresas representan el 95,4\% del total de las empresas, con un 39,8\% de personal ocupado (ver figura 1 ).

Figura 1. Estadísticas de las unidades económicas y personal ocupado del censo económico 2014

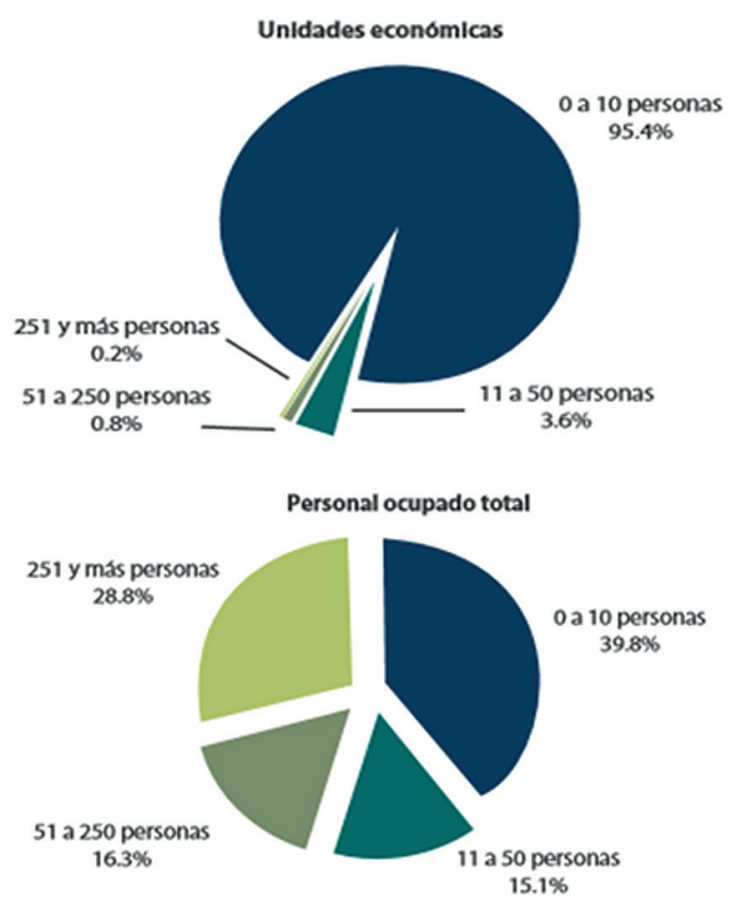

*El 95,4\% de unidades económicas corresponden a las microempresas, que emplean el 39,8\% de la población económicamente activa. Fuente: INEGI (2014).
En estas empresas, el sector manufacturero es uno de los más importantes debido a su capacidad para innovar y generar empleos: el 29,6\% de la población económicamente activa se encuentra en este sector, que representa el 97,8\% de unidades económicas. Concretamente, el estado de Tabasco tiene 1660 unidades económicas distribuidas en los 17 municipios que lo conforman, tal como puede observarse en la tabla 1 (INEGI, 2016a).

Tabla 1. Microempresas agroindustriales de estado de Tabasco

\begin{tabular}{|l|c|}
\hline \multicolumn{1}{|c|}{ MUNICIPIO } & MICRO \\
\hline Balancán & 46 \\
Cárdenas & 219 \\
Centla & 63 \\
Centro & 498 \\
Comalcalco & 163 \\
Cunduacán & 63 \\
Emiliano Zapata & 50 \\
Huimanguillo & 118 \\
Jalapa & 14 \\
Jalpa de Méndez & 59 \\
Jonuta & 24 \\
Macuspana & 83 \\
Nacajuca & 55 \\
Paraíso & 40 \\
Tacotalpa & 29 \\
Teapa & 63 \\
Tenosique & 73 \\
\hline Total & 1660 \\
\hline
\end{tabular}

Fuente: Elaboración propia con datos de INEGI (2016a). 
México tiene un gran número de empresas que se encuentran en el sector manufacturero, específicamente, en la industria alimentaria. Esta se enfoca en la elaboración, conservación y envasado de productos alimentarios derivados de la transformación de los productos de la agricultura, ganadería, riqueza forestal y pesca; de esa manera, son convertidos en productos para el consumo humano y de animales (INEGI, 2016a; Fideicomiso de Riesgo Compartido [Firco], 2017). Frente a este escenario, los gerentes deben diseñar e implementar estrategias que les permitan tener una gestión efectiva y fortalecer las actividades que realizan, pues en México se trata de un sector con mayor posibilidad de desarrollo y crecimiento. Entonces, al generar una mayor cantidad de empleos en el sector manufacturero, se beneficiará a la economía del país (INEGI, 2016b). El entorno cambiante en el que se encuentran las empresas demanda que tengan la capacidad de responder con éxito a las diferentes situaciones que se les presenten. Por ello, deben aprovechar las habilidades y capacidades de sus trabajadores, las nuevas tecnologías que pueden ser implementadas en sus procesos, y la innovación constante.

El objetivo principal de esta investigación es reportar los datos obtenidos en la tesis de investigación titulada Estrategias gerenciales de las micro y pequeñas empresas agroindustriales de Villahermosa, Tabasco, la cual fue realizada en la maestría en Administración y Dirección Estratégica en la Universidad Juárez Autónoma de Tabasco, México. En la investigación, se identificaron y analizaron las prácticas de gestión estratégica de las microempresas del sector agroindustrial de Villahermosa, Tabasco, con la finalidad de identificar si dichas organizaciones llevan a cabo el proceso de formulación, implementación y evaluación de las estrategias.

\section{Revisión literaria}

\subsection{Gestión estratégica}

Fernández (2006) menciona que «una gestión estratégica consiste en desarrollar estrategias competitivas para implementar las políticas y crear una estructura organizacional que sea favorable y conducente a una asignación de recursos que permita alcanzar con éxito esas estrategias» (p. 22). Siguiendo la misma línea, David (2011), define la "gestión estratégica» como la capacidad de una persona para formular, implementar y evaluar decisiones en la organización, con la finalidad de que se logren los objetivos que se hayan fijado para el éxito de la empresa.

La gestión estratégica también debe ser considerada como la habilidad de anticipar y gerenciar el cambio, que debe propiciar la creación permanente de estrategias que garanticen el futuro de la empresa: se debe considerar que, aunque se hayan diseñado e implementado estrategias óptimas en el pasado, estas no garantizan su éxito en el futuro. Por ello, se debe aprender constantemente y tener una visión del futuro deseado (Betancourt, 2006, p. 27). Por lo tanto, la gestión estratégica tiene como objetivo conducir a la empresa para que logre el éxito deseado; así, el gerente debe tener la capacidad de interpretar la información necesaria para tomar las decisiones que sean más convenientes, de tal manera que la empresa pueda mantenerse y avanzar en el mercado competitivo y agresivo en el que se encuentre (Brume, 2017).

La gestión estratégica debe realizarse de manera sistémica. De hecho, se conforma de tres etapas: la formulación, el despliegue o implementación, y la evaluación. Para llevar a cabo las tres etapas de la gestión estratégica, se debe realizar un análisis del entorno externo, de tal manera que se puedan identificar las 
oportunidades y amenazas; además, se debe analizar el entorno interno de la empresa, con la finalidad de determinar sus fortalezas y debilidades (Riaño-Casallas y García-Ubaque, 2016).

Prieto (2011) precisa que la gestión estratégica debe ser una herramienta vital para el análisis y evaluación de la situación actual de toda empresa, ya que le permite a la gerencia desarrollar las actividades de formulación y evaluación de estrategias alternativas para el funcionamiento de la organización. Esto produce la optimización de los recursos con los que cuenta. Además, la gestión estratégica debe permitir el diseño y ejecución de las políticas y procedimientos que sean acordes a los planes estratégicos de la organización. En ella, la base fundamental de una óptima gestión son las habilidades, talentos y aptitudes del liderazgo, así como las personas, las estrategias para realizar el trabajo, y la actitud que se asume ante los procesos y las habilidades que ha desarrollado la organización para funcionar de manera eficiente (Betancourt, 2006, p. 107; Bouso, 2010).

\subsubsection{Formulación de la estrategia}

En esta etapa de la gestión estratégica, es necesario realizar el análisis de los factores internos y externos que afectan a la organización, con el propósito de obtener información para formular la misión, visión, objetivos y metas, así como las estrategias, planes de acción y los indicadores de desempeño y logro, tanto para la empresa en general como para cada área que la integra (Sánchez, 2003). Asimismo, David (2011) menciona que en esta etapa se deben elaborar estrategias alternativas a seguir con las que la organización se vea beneficiada; así, cada gerente o líder es quien debe generar las estrategias anticipativas y adaptativas requeridas para sobrevivir y ser competitivos a corto, mediano y largo plazo. Además, las estrategias deben responder a la visión y la misión de la organización (Betancourt, 2006, p. 24).

Sin embargo, Riaño-Casallas y García-Ubaque (2016) mencionan que el proceso de formulación de la estrategia no debe depender solo de la alta dirección. Es recomendable que todos los integrantes de la organización participen en esta etapa, pues una estrategia bien formulada anticipará los cambios en el entorno y los movimientos de los competidores; además, permitirá prever la asignación correcta de los recursos, la cual estará basada en las capacidades y carencias internas de la organización.

\subsubsection{Implementación de la estrategia}

La implementación de la estrategia es la segunda etapa de la gestión estratégica. De acuerdo con David (2011), en esa etapa se deben realizar las siguientes actividades: a) establecer objetivos anuales, b) motivar a los empleados y asignar recursos para que las estrategias formuladas se ejecuten, c) desarrollar una cultura de apoyo estratégico, d) crear una estructura organizacional efectiva, e) preparar los presupuestos, f) vincular la remuneración con el desempeño organizacional, g) movilizar a los empleados y gerentes para poner en práctica las estrategias formuladas, y h) motivar a los empleados desde las gerencias.

\subsubsection{Evaluación de la estrategia}

La evaluación de la estrategia es la tercera etapa de la gestión estratégica. En ese momento, se debe analizar si las estrategias diseñadas e implementadas cumplen con lo esperado. Además, de acuerdo con David (2011), en esta etapa se puede realizar lo siguiente: a) determinar si las estrategias no funcionan bien, b) revisar los factores externos e internos que son las bases de las estrategias actuales, c) medir el desempeño, y d) tomar acciones correctivas. 


\subsection{Importancia de la agroindustria}

Las microempresas agroindustriales tienen un papel fundamental en los países de América Latina, ya que generan un número considerable de empleos para las actividades dentro de sus instalaciones, para las personas que proveen los materiales necesarios para el producto final y para los que realizan la distribución de los productos. De igual manera, estas empresas generan beneficios económicos, que se extienden a las regiones vulnerables. Además, permiten mejorar la seguridad alimentaria al suministrar productos de la canasta básica (Organización de las Naciones Unidas para la Alimentación y la Agricultura [FAO], 2004).

El sector de la agroindustria produce numerosos beneficios, como la generación de empleo; la prolongación de vida útil de los productos; y el reconocimiento del país en diferentes lugares del mundo, ya que los productos elaborados por las agroindustrias mexicanas se distribuyen y comercializan a nivel local, nacional e internacional, con lo que contribuyen al desarrollo económico regional y nacional. La globalización permite el acceso a nuevos mercados, lo que se convierte en un reto importante para las empresas locales. Por este motivo, los gerentes de las agroindustrias deben tener la habilidad y capacidad para reaccionar de manera rápida y eficaz ante los cambios. Entre ellos, se encuentra la entrada de competidores extranjeros al mercado nacional, que implica el diseńo de estrategias con las que puedan lograr diferenciarse y proporcionar más valor al consumidor con los productos que elaboran (Montealegre, Delgado \& Cubillos, 2015, citados por Suárez, 2019).

Ya que las agroindustrias se desenvuelven en un mercado que cambia constantemente, los gestores deben evaluar sus empresas, es decir, identificar sus fortalezas, debilidades, oportunidades y amenazas. Además, deben analizar sus habilidades gerenciales, ya que estas constituyen el crecimiento y permanencia de las empresas a nivel nacional e internacional (Lara, 2017, Méndez, 2007, citados por Melo y Fonseca, 2014).

\subsection{Microempresas agroindustriales mexicanas}

Las microempresas agroindustriales se han convertido en unidades de gran importancia para la economía de México, ya que aportan el 11,6\% de unidades económicas y generan empleos para el $23,5 \%$ de la población económicamente activa (más de seis mil millones de personas en el país) (INEGI, 2014). De hecho, México ha sido calificado como líder en el sector agroindustrial, debido a su capacidad para entender la producción, industrialización y comercialización de los productos forestales, agropecuarios y biológicos; en el sector, se procura la durabilidad de los productos que son más perecederos (Serrano, 2017).

Más allá de los aspectos mencionados, el sector agroindustrial mexicano todavía requiere de recursos que le permitan tener mayor crecimiento y desarrollo. Por ello, se debe procurar la innovación constante, la capacitación de los trabajadores y la inversión en tecnologías que beneficien a las empresas del sector. El INEGI (2016b) desarrolló una línea de investigación llamada «Demografía económica», la cual tuvo como principal objetivo identificar los indicadores que estuvieran relacionados con el nivel de supervivencia, muerte y años de vida de las empresas en México. En el estudio, se encontró que las microempresas tienen un alto índice de mortalidad; en cambio, las pequeñas, medianas y grandes empresas tienen mayor esperanza de vida (ver tabla 2).

Otra investigación importante fue la publicada por el INEGI a través del Directorio Estadístico Nacional de Unidades Económicas (en adelante, DENUE) 
(INEGI, 2016a), en el que se comparó la mortandad y nacimiento de empresas en un periodo de cinco años (desde 2010 hasta 2015). En esa investigación, se encontró que, en el año 2010, se registraron 4,3 millones de empresas, mientras que 1,6 millones de establecimientos murieron. Además, en el año 2015, se registraron 4,9 millones de establecimientos (2,2 millones fueron de nueva creación), mientras que la tasa de mortalidad de las microempresas fue de $34,3 \%$; asimismo, en el 2015, se registró que 46.4\% de las microempresas fueron de nueva creación en los sectores económicos que se observan en la tabla 3 (Suárez, 2019).

Tabla 2. Probabilidad de muerte y esperanza de vida en el primer ańo para negocios de 0-100 personas ocupadas

\begin{tabular}{|l|c|c|c|}
\hline \multirow{3}{*}{ Micro } & $\begin{array}{c}\text { Tamańo de } \\
\text { los negocios } \\
\text { (personas } \\
\text { ocupadas) }\end{array}$ & $\begin{array}{c}\text { Probabilidad } \\
\text { de muerte al } \\
\text { primer ańo de } \\
\text { vida }\end{array}$ & $\begin{array}{c}\text { Esperanza de } \\
\text { vida al nacer } \\
\text { (años futuros } \\
\text { de vida) }\end{array}$ \\
\hline \multirow{3}{*}{ Pequeña } & $3-2$ & 0,38 & 6,9 \\
& $11-15$ & 0,32 & 8,4 \\
& $16-20$ & 0,17 & 15,0 \\
\hline Grande & $21-30$ & 0,18 & 20,7 \\
\hline
\end{tabular}

Fuente: INEGI (2016b).

Tabla 3. Muertes y nacimientos de empresas en el periodo 2010-2015

\begin{tabular}{|l|c|c|}
\hline \multirow{2}{*}{ SECTOR } & MUERTES & NACIMIENTOS \\
\cline { 2 - 3 } & $\mathbf{2 0 1 0 - 2 0 1 5}$ & $\mathbf{2 0 1 0 - 2 0 1 5}$ \\
\hline Comercio & $17,4 \%$ & $21,7 \%$ \\
Servicio & $3,5 \%$ & $5,3 \%$ \\
Manufacturas & $2,3 \%$ & $\mathbf{2 , 8} \%$ \\
\hline
\end{tabular}

Fuente: Elaboración propia con datos de INEGI (2016b).
Los indicadores de demografía económica se comportan de manera diferente dependiendo del sector de la actividad. Concretamente, se puede observar la probabilidad de supervivencia y esperanza de vida en la tabla 4 (Suárez, 2019).

Tabla 4. Probabilidad de supervivencia y esperanza de vida por sector de actividad en el primer ańo de vida de las empresas

\begin{tabular}{|l|c|c|}
\hline \multicolumn{1}{|c|}{ Sector } & $\begin{array}{c}\text { Probabilidad de } \\
\text { supervivencia durante } \\
\text { el primer ańo de vida }\end{array}$ & $\begin{array}{c}\text { Esperanza de vida } \\
\text { al nacer (años de } \\
\text { vida futura) }\end{array}$ \\
\hline Manufactura & 0,68 & 9,5 \\
Comercio & 0,62 & 6,6 \\
$\begin{array}{l}\text { Servicios privados } \\
\text { no financieros }\end{array}$ & 0,64 & 8,0 \\
Los tres sectores & 0,64 & 7,7 \\
\hline
\end{tabular}

Fuente: INEGI (2016b).

Las microempresas agroindustriales son vitales para la economía mexicana. Por ello, los gerentes deben gestionar sus recursos para lograr su permanencia y desarrollo en el mercado por medio del uso de nuevas tecnologías e inversiones, entre otras estrategias: las agroindustrias aportan cada vez más unidades económicas que propician la generación de empleos. Además, cada región tiene condiciones favorables para las actividades que se realizan en el sector, y, en los últimos años, las empresas han logrado obtener mayor presencia en el mercado regional, nacional e internacional.

\section{Metodología}

\subsection{Participantes}

De acuerdo con el DENUE (INEGI, 2016a), el municipio de Centro en el estado de Tabasco tiene registradas 498 microempresas dentro del sector agroindustrial. Entonces, para determinar el número de encuestas en las microempresas, se realizó un muestreo 
probabilístico, con un nivel de confianza del 95\% y un margen de error del 5\%. Posteriormente, se procedió a realizar un muestreo intencional, a través del cual se pueden seleccionar algunos casos para analizarlos y obtener la información necesaria para la investigación (Hernández, Fernández \& Baptista, 2014). Por ello, se examinó el DENUE (INEGI, 2016a), en el que se pueden encontrar los datos necesarios para identificar a las microempresas agroindustriales de Villahermosa, Tabasco, así como el nombre de la empresa, el número de empleados, la dirección y el teléfono, entre otros datos.

Sobre la base de la información obtenida a través del DENUE, se seleccionaron las empresas dedicadas a diferentes actividades (ver tabla 5). Estas fueron elegidas por la cantidad de empresas dedicadas a la misma actividad, así como la cercanía y seguridad de la zona en las que se localizan. En total, se obtuvieron 194 microempresas del sector agroindustrial de Villahermosa, Tabasco.

Tabla 5. Actividad de la empresa

\begin{tabular}{|l|c|c|}
\hline \multicolumn{1}{|c|}{ Actividad } & Micro & $\%$ \\
\hline $\begin{array}{l}\text { Elaboración de concentrados, polvos, jara- } \\
\text { bes y esencias de sabor para bebidas }\end{array}$ & 1 &, 5 \\
$\begin{array}{l}\text { Elaboración de derivados y fermentos } \\
\text { lácteos }\end{array}$ & 3 & 1,5 \\
Elaboración de botanas & 8 & 4,1 \\
$\begin{array}{l}\text { Elaboración de chocolate y productos de } \\
\text { chocolate }\end{array}$ & 1 &, 5 \\
Elaboración de galletas y pastas para sopa & 2 & 1,0 \\
Elaboración de helados y paletas & 31 & 16,0 \\
Elaboración de condimentos y aderezos & 2 & 1,0 \\
Elaboración de tortillas de maíz y molienda & 64 & 33,0 \\
de nixtamal & 82 & 42,3 \\
Panificación tradicional & 194 & 100,0 \\
\hline Total & & \\
\hline
\end{tabular}

Fuente: Elaboración propia con datos del DENUE (INEGI, 2016a).

\subsection{Instrumento}

El instrumento fue construido sobre la base de tres investigaciones realizadas anteriormente: 1) el cuestionario de diagnóstico empresarial basado en el modelo de la Fundación Europea para la Gestión de la Calidad y Excelencia (EFQM), implementado en una investigación acerca del proceso de autoevaluación de una pyme por Tarí, López y Molina (2007); 2) el instrumento aplicado a la gerencia del sector metalmecánico de la pyme de Acarigua para conocer las estrategias gerenciales basadas en la calidad de gestión de la pequeña y mediana empresa, implementado por Urdaneta (2013); y 3) el instrumento aplicado en una investigación realizada por Daboin y Romero (2010), que fue implementado para identificar las estrategias gerenciales y de motivación para el logro en los empleados de empresas manufactureras ubicadas en Maracaibo, Zulia.

El cuestionario se organizó en dos partes. La primera sección está diseñada para recolectar datos acerca de la actividad de la empresa; el número de empleados con los que cuentan; el puesto ocupado por la persona encuestada; y la información sociodemográfica de los participantes, tal como la edad, la antigüedad en la empresa, el género y el estado civil. La segunda parte del instrumento se diseñó para medir la gestión estratégica a través de tres dimensiones: formulación de la estrategia, implementación de la estrategia y evaluación de la estrategia; se consideró el sentido de cada frase poniendo especial interés en la claridad de la redacción para poder construir los ítems del cuestionario y facilitar su comprensión para los gerentes encuestados de las microempresas agroindustriales.

El instrumento tiene una variable a investigar denominada «estrategias gerenciales». Estas corresponden a las acciones gerenciales que le permitirán a la empresa alcanzar las metas y objetivos establecidos, considerando 
los recursos y capacidades de la misma, con el fin de obtener beneficios de las fortalezas internas y oportunidades externas para disminuir las debilidades internas y enfrentar con éxito las amenazas externas (Melo \& Fonseca, 2014; Noguera, Barbosa \& Castro, 2014; Marín, 2013; Urdaneta, 2013; Rubio, 2012; David, 2011; Elizalde, 2011; Chiavenato, 2007).

El cuestionario está constituido por 36 ítems. Estos se encuentran agrupados en tres dimensiones: formulación de la estrategia, implementación de la estrategia y evaluación de la estrategia.

- Dimensión de formulación de la estrategia: consiste en el diseño de la misión y visión de la empresa. Establece objetivos a largo plazo. Además, detecta las oportunidades y amenazas del entorno, así como las fortalezas y debilidades de la empresa, para poder establecer acciones a seguir con las que la organización se vea beneficiada. Esta dimensión contiene 21 reactivos $(1,2,3,4,5,6,7,8,9,10$, $11,12,13,14,15,16,17,18,19,20,21)$.

- Dimensión de implementación de la estrategia: consiste en poner los planes y estrategias en acción, movilizando a todo el personal para alcanzar los objetivos de la empresa. Esta dimensión contiene 10 reactivos $(22,23,24,25,26,27,28,29,30,31)$.

- Dimensión de evaluación de la estrategia: consiste en conocer y analizar si las acciones están dirigiendo a la organización a lograr los objetivos; además, se basa en obtener información para formular estrategias que mejoren el futuro de la empresa. Esta dimensión contiene 5 reactivos $(32,33,34,35,36)$.

El cuestionario presenta un encabezado genérico («La empresa...»), con el cual se pretende que el gerente responda qué tanto se realiza la actividad indicada en su organización. Las respuestas a los ítems se realizaron sobre la base de una escala Likert de cinco puntos ( 1 = nunca, 2 = casi nunca, 3 = algunas veces, 4 = casi siempre, 5 = siempre).

\subsection{Confiabilidad y validez del instrumento}

Para llevar a cabo la validación del instrumento que permitiera medir la gestión estratégica de las microempresas seleccionadas y saber con certeza si los datos eran idóneos para el análisis, se utilizó un análisis factorial exploratorio con el método de máxima verosimilitud y rotación Oblimin directo. Por eso, se calculó la medida de adecuación de la muestra de Kaiser Meyer Olkin $(K M O)$, que fue de .920; y la prueba de esfericidad de Bartlett, que presentó valores de $\left(\mathrm{X}^{2}=8909,066\right.$, gl= 630, $\mathrm{p} \leq .000)$. Con estos datos, el análisis confirmó la estructura factorial explicando el 56,74\% de la varianza, lo que indica que la matriz de correlaciones es apta para la factorización (Moral, 2011).

Este análisis factorial exploratorio permitió realizar la validación del constructo con tres factores: formulación de la estrategia, implementación de la estrategia y evaluación de la estrategia. El modelo determinó tres factores con cargas factoriales arriba de ,3, excepto en el ítem 31 (ver tabla 6). Esto indica una estructura aceptable (Moral, 2011).

La confiabilidad que se reporta para los datos que se presentan es de un alfa de Cronbach de 0,967, lo que se considera como valores de confiabilidad aceptables del instrumento a aplicar en la investigación (García, 2011; Hernández et al., 2014). El instrumento reporta un alfa de Cronbach para cada una de las dimensiones del cuestionario: la dimensión de formulación de la estrategia reporta un 0,968 de confiabilidad; la dimensión implementación de la estrategia, un 0.893 de confiabilidad; $y$, por último, la dimensión de evaluación de la estrategia, un 0.774 de confiabilidad. Se tratan de valores aceptables para el cuestionario utilizado. 


\section{Tabla 6. Análisis factorial con rotación Oblimin directo}

\begin{tabular}{|c|c|c|c|}
\hline Ítems & Formulación & Implementación & Evaluación \\
\hline $\begin{array}{l}\text { 1. Analiza indicadores económicos, demográficos, políticos y tecnológicos para detec- } \\
\text { tar oportunidades en el entorno. }\end{array}$ & 1,102 &,- 080 &,- 149 \\
\hline $\begin{array}{l}\text { 2. Analiza indicadores económicos, demográficos, políticos y tecnológicos para detec- } \\
\text { tar amenazas en el entorno. }\end{array}$ & 1,099 &,- 074 &,- 152 \\
\hline $\begin{array}{l}\text { 3. Analiza los factores que hacen diferente a su empresa con respecto a otras del mismo } \\
\text { sector. }\end{array}$ & ,990 &, 000 &,- 139 \\
\hline $\begin{array}{l}\text { 4. Analiza las áreas y actividades que debe mejorar para realizar una mejor gestión de } \\
\text { las mismas. }\end{array}$ & ,963 &,- 006 &,- 175 \\
\hline $\begin{array}{l}\text { 5. Realiza estudios de mercado para conocer las necesidades y preferencias de los con- } \\
\text { sumidores. }\end{array}$ & ,689 &, 129 & 020 \\
\hline 6. Utiliza herramientas de mercadotecnia para promocionar los productos. & ,671 & ,088 & 079 \\
\hline 7. Desarrolla estrategias para ampliar el mercado de clientes. & ,643 & 021 & ,133 \\
\hline 8. Tiene por escrito la misión y la visión. & ,641 & 249 & 001 \\
\hline $\begin{array}{l}\text { 9. Identifica el segmento de mercado en los que sus productos y/o servicios son más } \\
\text { aceptados. }\end{array}$ & ,602 &,- 057 & 066 \\
\hline 10. Desarrolla estrategias para diversificar los productos que ofrece. & ,600 & ,008 &, 113 \\
\hline $\begin{array}{l}\text { 11. Utiliza aplicaciones informáticas para la difusión del conocimiento entre sus depar- } \\
\text { tamentos. }\end{array}$ &, 595 &, 132 & ,078 \\
\hline 12. Tiene un organigrama escrito y lo da a conocer a todos los integrantes de la empresa. &, 595 & ,262 & 013 \\
\hline $\begin{array}{l}\text { 13. Tiene manuales de procedimientos y los da a conocer a todos los integrantes de la } \\
\text { empresa. }\end{array}$ &, 588 &,- 053 & ,238 \\
\hline 14. Cuenta con los recursos necesarios para realizar sus operaciones. &, 584 &,- 043 & 076 \\
\hline $\begin{array}{l}\text { 15. Tiene manuales de organización y los da a conocer a todos los integrantes de la } \\
\text { empresa. }\end{array}$ &, 570 &, 106 &, 161 \\
\hline $\begin{array}{l}\text { 16. Implementa nuevas aplicaciones informáticas, como la presencia en internet, comer- } \\
\text { cio electrónico y páginas web. }\end{array}$ &, 562 &, 189 & 013 \\
\hline 17. Utiliza aplicaciones informáticas para el control de inventarios/compras. &, 542 & ,292 & 092 \\
\hline 18. Introduce nuevos productos al mercado. &, 534 &, 125 & 067 \\
\hline $\begin{array}{l}\text { 19. Formula estrategias de supervisión para las actividades que se realizan en cada área } \\
\text { de la empresa. }\end{array}$ &, 529 &,- 165 & ,232 \\
\hline 20. Coloca al personal en cada área de trabajo de acuerdo a sus capacidades y habilidades. & ,497 &, 105 & 004 \\
\hline 21. Lleva a cabo un control para medir las operaciones realizadas por los empleados. & ,487 &, 112 &, 157 \\
\hline 22. Gestiona recursos para conocer las nuevas tecnologías que usa su sector. & ,414 & ,406 &, 177 \\
\hline 23. Comunica a todas sus áreas las actividades que deben realizarse. & ,377 &, 058 &, 253 \\
\hline 24. Realiza prácticas para que el personal se sienta satisfecho con su trabajo. & 017 & 914 & ,271 \\
\hline 25. Fomenta prácticas que motivan al personal para realizar mejor su trabajo. & 019 & ,907 & ,293 \\
\hline 26. Existe delegación de autoridad en su organización. &, 167 & ,625 &,- 199 \\
\hline
\end{tabular}




\begin{tabular}{|l|c|c|c|}
\hline \multicolumn{1}{|c|}{ Ítems } & Formulación & Implementación & Evaluación \\
\hline 27. Planifica sus actividades enfocadas en el logro de los objetivos. &, 305 &, 546 &,- 225 \\
\hline 28. La planificación se lleva a cabo en su organización. &, 434 &, 466 &,- 176 \\
\hline 29. Se aplican acciones correctivas en la empresa. &, 269 &, 407 &,- 031 \\
\hline $\begin{array}{l}\text { 30. Se asegura de que se logren las metas contempladas en la planificación. } \\
\text { grupos. }\end{array}$ &, 275 &, 345 &, 080 \\
\hline $\begin{array}{l}\text { 31. Como estrategia, se agrupa con otras empresas del mismo sector para compras en } \\
\text { 32. Verifica que los empleados respeten las normas de seguridad en el trabajo. }\end{array}$ &, 112 &, 092 \\
\hline $\begin{array}{l}\text { 33. Como estrategia, se asegura de que todo el personal cumpla con su código de ética } \\
\text { empresarial. }\end{array}$ &, 005 &, 007 &, 141 \\
\hline 34. Pone en práctica los valores institucionales que tiene establecidos. &, 077 &, 956 \\
\hline 35. Gestiona sus recursos para capacitar a los empleados y directivos. &, 235 &,- 258 \\
\hline 36. Supervisa las actividades realizadas por los trabajadores. &, 214 &,- 763 \\
\hline
\end{tabular}

Fuente: Suárez (2019).

\subsection{Procedimiento}

Los datos obtenidos se recolectaron con lápiz y papel dentro de la empresa. Además, se respondieron dudas en el lugar de aplicación; y se garantizó a los encuestados confidencialidad de los datos proporcionados, lo que permitió que todos los gerentes de las empresas seleccionadas en la muestra tuvieran la confianza y accesibilidad para responder el cuestionario aplicado.

\section{Resultados}

El estudio se realizó a 194 gerentes de microempresas agroindustriales de Villahermosa, Tabasco, donde el $42,3 \%$ se dedican a la panificación tradicional. De los 194 gerentes, el 52,6\% (102) fueron mujeres, mientras que el 47,9\% (92) fueron hombres, con un rango de edad de 31 a 36 años $(36,1 \%)$.

En la tabla 7, se puede notar que los gerentes de las microempresas investigadas sí llevan a cabo la evaluación de las estrategias que implementan, de tal manera que puedan realizar las acciones correctivas necesarias en su proceso de gestión. Sin embargo, la etapa que menos realizan es la formulación de la estrategia, ya que, por lo general, los gerentes implementan estrategias de manera empírica; es decir, ejecutan acciones dependiendo de la situación en la que se encuentren.

Tabla 7. Gestión estratégica de las microempresas

\begin{tabular}{|l|c|c|c|c|c|}
\hline & N & Mínimo & Máximo & Media & DS \\
\hline $\begin{array}{l}\text { Formulación de la } \\
\text { estrategia }\end{array}$ & 194 & 1 & 5 & 3,29 & 1,029 \\
\hline $\begin{array}{l}\text { Implementación de } \\
\text { la estrategia }\end{array}$ & 194 & 2 & 5 & 3,57 &, 706 \\
\hline $\begin{array}{l}\text { Evaluación de la } \\
\text { estrategia }\end{array}$ & 194 & 3 & 5 & 4,11 &, 563 \\
\hline N válido (por lista) & 194 & & & & \\
\hline
\end{tabular}

Fuente: Suárez (2019).

En la dimensión de formulación de la estrategia (ver tabla 8), se encontró que las microempresas consideran que tienen los recursos necesarios para realizar sus actividades. De esta manera, pueden formular sus estrategias sin preocuparse por tener recursos limitados, tal como la materia prima necesaria para elaborar sus productos, maquinarias y personal, entre otros. 
Tabla 8. Análisis descriptivo de la dimensión de formulación de la estrategia de las microempresas

\begin{tabular}{|c|c|c|c|c|c|}
\hline Reactivo & $\mathrm{N}$ & Mínimo & Máximo & Media & DS \\
\hline $\begin{array}{l}\text { 1. Analiza indicadores económicos, demográficos, políticos y tecnológicos para detec- } \\
\text { tar amenazas en el entorno. }\end{array}$ & 194 & 1 & 5 & 3,01 & 1,236 \\
\hline $\begin{array}{l}\text { 2. Analiza indicadores económicos, demográficos, políticos y tecnológicos para detec- } \\
\text { tar oportunidades en el entorno. }\end{array}$ & 194 & 1 & 5 & 3,02 & 1,232 \\
\hline $\begin{array}{l}\text { 3. Analiza los factores que hacen diferente a su empresa con respecto a otras del mismo } \\
\text { sector. }\end{array}$ & 194 & 1 & 5 & 3,15 & 1,278 \\
\hline $\begin{array}{l}\text { 4. Analiza las áreas y actividades que debe mejorar para realizar una mejor gestión de } \\
\text { las mismas. }\end{array}$ & 194 & 1 & 5 & 3,24 & 1,261 \\
\hline 5. Tiene por escrito la misión y la visión. & 194 & 1 & 5 & 2,55 & 1,731 \\
\hline 6. Tiene un organigrama escrito y lo da a conocer a todos los integrantes de la empresa. & 194 & 1 & 5 & 2,62 & 1,739 \\
\hline $\begin{array}{l}\text { 7. Tiene manuales de procedimientos y los da a conocer a todos los integrantes de la } \\
\text { empresa. }\end{array}$ & 194 & 1 & 5 & 3,77 & 1,370 \\
\hline $\begin{array}{l}\text { 8. Tiene manuales de organización y los da a conocer a todos los integrantes de la } \\
\text { empresa. }\end{array}$ & 194 & 1 & 5 & 3,57 & 1,488 \\
\hline 9. Coloca al personal en cada área de trabajo de acuerdo a sus capacidades y habilidades. & 194 & 1 & 5 & 4,30 & ,805 \\
\hline 10. Cuenta con los recursos necesarios para realizar sus operaciones. & 194 & 3 & 5 & 4,59 &, 553 \\
\hline $\begin{array}{l}\text { 11. Formula estrategias de supervisión para las actividades que se realizan en cada área } \\
\text { de la empresa. }\end{array}$ & 194 & 1 & 5 & 3,66 & 1,076 \\
\hline 12. Lleva a cabo un control para medir las operaciones realizadas por los empleados. & 194 & 1 & 5 & 3,82 & 1,048 \\
\hline $\begin{array}{l}\text { 13. Realiza estudios de mercado para conocer las necesidades y preferencias de los con- } \\
\text { sumidores. }\end{array}$ & 194 & 1 & 5 & 2,68 & 1,313 \\
\hline $\begin{array}{l}\text { 14. Identifica el segmento de mercado en los que sus productos y/o servicios son más } \\
\text { aceptados. }\end{array}$ & 194 & 1 & 5 & 3,78 & ,984 \\
\hline 15. Utiliza herramientas de mercadotecnia para promocionar los productos. & 194 & 1 & 5 & 3,20 & 1,480 \\
\hline 16. Desarrolla estrategias para ampliar el mercado de clientes. & 194 & 1 & 5 & 3,30 & 1,325 \\
\hline 17. Desarrolla estrategias para diversificar los productos que ofrece. & 194 & 1 & 5 & 3,16 & 1,385 \\
\hline $\begin{array}{l}\text { 18. Implementa nuevas aplicaciones informáticas, como la presencia en internet, comer- } \\
\text { cio electrónico y páginas web. }\end{array}$ & 194 & 1 & 5 & 2,57 & 1,634 \\
\hline $\begin{array}{l}\text { 19. Utiliza aplicaciones informáticas para la difusión del conocimiento entre sus depar- } \\
\text { tamentos. }\end{array}$ & 194 & 1 & 5 & 3,26 & 1,439 \\
\hline 20. Utiliza aplicaciones informáticas para el control de inventarios/compras. & 194 & 1 & 5 & 2,89 & 1,721 \\
\hline 21. Introduce nuevos productos al mercado. & 194 & 1 & 5 & 2,99 & 1,219 \\
\hline $\mathrm{N}$ válido (por lista) & 194 & & & & \\
\hline
\end{tabular}

Fuente: Suárez (2019). 
Sin embargo, las microempresas realizan una gestión empírica, pues no tienen definida su filosofía organizacional, tal como la misión, visión y valores; esto puede afectar el logro de los objetivos y metas establecidas, así como las actividades que realizan.

La segunda etapa de la gestión estratégica es la implementación de la estrategia (ver tabla 9), en la que se encontró que los gerentes de las microempresas se aseguran de que todo el personal conozca a detalle las actividades que deben realizarse para lograr la estrategia que ha sido diseńada para alcanzar los objetivos de la empresa. Asimismo, se pudo comprobar que los gerentes de las microempresas agroindustriales no realizan alianzas estratégicas con otras empresas de su mismo sector; esto puede ser una estrategia que les brinde algunas ventajas, pues se pueden realizar compras por volumen, con lo que se reducen los costos de producción.

Tabla 9. Análisis descriptivo de la dimensión de implementación de la estrategia de las microempresas

\begin{tabular}{|c|c|c|c|c|c|}
\hline Reactivo & $\mathrm{N}$ & Mínimo & Máximo & Media & DS \\
\hline 22. La planificación se lleva a cabo en su organización. & 194 & 1 & 5 & 4,09 & 1,009 \\
\hline 23. Planifica sus actividades enfocadas en el logro de los objetivos. & 194 & 1 & 5 & 3,75 & 1,352 \\
\hline 24. Comunica a todas sus áreas las actividades que deben realizarse. & 194 & 1 & 5 & 4,35 & ,675 \\
\hline 25. Existe delegación de autoridad en su organización. & 194 & 1 & 5 & 3,73 & 1,320 \\
\hline 26. Fomenta prácticas que motivan al personal para realizar mejor su trabajo. & 194 & 2 & 5 & 3,91 &, 721 \\
\hline 27. Realiza prácticas para que el personal se sienta satisfecho con su trabajo. & 194 & 2 & 5 & 3,89 & ,708 \\
\hline 28. Logra las metas contempladas en la planificación. & 194 & 3 & 5 & 4,30 & ,662 \\
\hline 29. Se aplican acciones correctivas en la empresa. & 194 & 1 & 5 & 4,18 & 1,015 \\
\hline 30. Gestiona recursos para conocer las nuevas tecnologías que usa su sector. & 194 & 1 & 5 & 2,23 & 1,396 \\
\hline $\begin{array}{l}\text { 31. Como estrategia, se agrupa con otras empresas del mismo sector para compras en } \\
\text { grupos. }\end{array}$ & 194 & 1 & 4 & 1,27 & ,677 \\
\hline $\mathrm{N}$ válido (por lista) & 194 & & & & \\
\hline
\end{tabular}

Fuente: Suárez (2019).

Tabla 10. Análisis descriptivo de la dimensión de evaluación de la estrategia de las microempresas

\begin{tabular}{|c|c|c|c|c|c|}
\hline Reactivo & $\mathrm{N}$ & Mínimo & Máximo & Media & DS \\
\hline 32. Supervisa las actividades realizadas por los trabajadores. & 194 & 2 & 5 & 4,51 &, 596 \\
\hline $\begin{array}{l}\text { 33. Como estrategia, se asegura de que todo el personal cumpla con su código de ética } \\
\text { empresarial. }\end{array}$ & 194 & 3 & 5 & 4,32 &, 532 \\
\hline 34. Verifica que los empleados respeten las normas de seguridad en el trabajo. & 194 & 3 & 5 & 4,35 &, 530 \\
\hline 35. Gestiona sus recursos para capacitar a los empleados y directivos. & 194 & 1 & 5 & 3,13 & 1,328 \\
\hline 36. Pone en práctica los valores institucionales que tiene establecidos. & 194 & 2 & 5 & 4,25 & ,603 \\
\hline N válido (por lista) & 194 & & & & \\
\hline
\end{tabular}

Fuente: Suárez (2019). 
Por último, en la etapa de la evaluación de la estrategia (ver tabla 10), se encontró que los gerentes de las microempresas realizan la supervisión de las actividades que fueron diseñadas y ejecutadas por los trabajadores. Esto les permite verificar que las acciones se realizan de acuerdo a lo planeado y, cuando sea el caso, realizar las acciones correctivas necesarias para el logro de los objetivos que la empresa se ha fijado. Sin embargo, se encontró que los gerentes de las microempresas no gestionan sus recursos para capacitar a sus empleados y directivos. Esto puede trascender a la productividad de los mismos, ya que la capacitación es vital para que los trabajadores desarrollen sus habilidades y competencias, pues les permitirá realizar de manera óptima sus actividades; así, la empresa se puede ver beneficiada, ya que puede ser más competitiva y desarrollarse en el mercado en el que se encuentra.

\section{Conclusiones}

La presencia de las microempresas en el mundo es de suma importancia: son unidades representativas en el ámbito económico y social, debido a la cantidad de empleos que generan. Frente a ello, la investigación permitió identificar la manera en que los gerentes de las microempresas del sector agroindustrial de Villahermosa, Tabasco, realizan su gestión estratégica. Los resultados brindan evidencia de que los gerentes de dichas empresas implementan estrategias para lograr sus objetivos de manera empírica, ya que no planifican las actividades que deben realizarse, sino que actúan dependiendo de la situación a la que se enfrentan. Aun así, los gerentes de estas empresas realizan un análisis y evaluación para determinar si las acciones que implementan los acerca a sus objetivos.

Lo anterior coincide con el estudio realizado por Melo y Fonseca (2014), en el cual se concluye que solo el $61 \%$ de la gerencia de las mipymes agroindustriales del departamento de Boyacá, Colombia formulan las estrategias que deben implementarse, pero no llevan a cabo la planificación de las actividades que deben realizarse para alcanzar un crecimiento y desarrollo a largo plazo. De igual manera, en el estudio realizado por Montealegre et al. (2015; citados por Suárez, 2019), se encontró que el $67 \%$ de las empresas agroindustriales del departamento del Tolima, Colombia, no tienen procesos gerenciales definidos, por lo que no realizan la gestión estratégica adecuada que fomente el crecimiento y permanencia de las mismas.

En la etapa de la formulación de la estrategia, se concluye que los gerentes de las microempresas consideran que tienen los recursos necesarios para realizar sus actividades, tal como la materia prima, la maquinaria y el personal, entre otros recursos. Sin embargo, se evidenció que estas empresas no han establecido su filosofía organizacional, lo que puede influir en su gestión: no tienen definido el camino a seguir, a pesar de que una visión y misión definidas le permite a la empresa identificar en dónde se encuentra y hacia dónde debe dirigirse; además, la visión y la misión permiten que la empresa sepa su propósito y las acciones a realizar para ser competitiva, con lo que pueda lograr los resultados planeados (Thompson, Peteraf, Gamble, \& Strickland, 2015).

Los resultados evidencian que, en la etapa de la implementación de la estrategia, los gerentes de las microempresas no realizan una planificación formal sobre las estrategias que deben implementarse para lograr sus objetivos. No obstante, cuando los gerentes analizan la situación de su empresa, deciden las acciones que se van a realizar, además de que se aseguran de que todo el personal conozca las actividades que se van a ejecutar. Estos resultados coinciden con la investigación de Urdaneta (2013), en la que se evidenció que el 55\% de los gerentes estudiados consideraban que en sus 
empresas existe un proceso comunicacional durante la toma de decisiones y, aunque existe deficiencias en él, se está trabajando para mejorar este aspecto dentro de la organización.

Con respecto a la etapa de la evaluación de la estrategia, los resultados permiten concluir que los gerentes de las microempresas agroindustriales realizan la supervisión de las actividades que ejecutan los trabajadores, con la finalidad de verificar que estas se realicen de acuerdo a lo que se ha planeado y, cuando sea el caso, efectuar las acciones correctivas que sean necesarias para lograr los objetivos de la empresa. Sin embargo, los gerentes de las microempresas del sector agroindustrial no gestionan la capacitación para sus trabajadores. Esto puede afectar la productividad de los mismos, pues la capacitación es necesaria para que los trabajadores desarrollen sus habilidades y competencias, a través de la adquisición de nuevos conocimientos con los que puedan mejorar la ejecución de sus actividades; así, se propiciaría que la empresa sea más rentable.

Por lo señalado, resulta indispensable que los gerentes de estas empresas realicen un diagnóstico de la situación en la que se encuentran, con el fin de que detecten sus deficiencias y que apliquen las medidas necesarias para corregirlas, con lo que puedan optimizar su funcionamiento. En definitiva, es necesario realizar más investigaciones sobre la gestión estratégica de las microempresas agroindustriales de Villahermosa, Tabasco, de manera específica, en cada empresa contemplada para este estudio y en otros tamaños de unidades económicas. Además, se deben considerar otras variables que no han sido tomadas en esta investigación con el fin de generar mayor evidencia de la problemática de este sector y proponer recomendaciones para que las empresas puedan realizar una gestión estratégica adecuada que les permita obtener beneficios futuros.

\section{Referencias}

Betancourt, J. R. (2006). Gestión estratégica: Navegando hacia el cuarto paradigma. Porlamar: Eumed. Recuperado de http://www.eumed.net/libros-gratis/2006c/220/index. htm [Consulta: 16 de noviembre de 2020].

Bouso, A. I. (2010, 8-10 de diciembre). Análisis de la gestión estratégica como herramienta para el mejoramiento continuo en la educación superior [Presentación en coloquio]. X Coloquio Internacional sobre Gestión Universitaria en América del Sur, Mar de Plata, Argentina. Recuperado de https://www.google.com.mx/url?sa=t\&source=web \&rct=j\&url=https://repositorio.ufsc.br/bitstream/handle/123456789/96748/BOUSO.pdf\%3Fsequence\%3D $18 v e d=0$ ahUKEwjO3sPA8c7XAhVE6GMKHZopA 10QFggtMAA\&usg=AOvVaw1Ob1o3J6_Dh4B0tLXcDCo [Consulta: 17 de noviembre de 2020].

Brume, M. J. (2017). Gestión estratégica como herramienta para promover la competitividad de las empresas del sector logístico del departamento del Atlántico, Colombia. Espacios, 38(91), 20-34. Recuperado de https:// www.revistaespacios.com/a17v38n51/a17v38n51p20. pdf [Consulta: 16 de noviembre de 2020].

Chiavenato, I. (2007). Introducción a la teoría general de la administración. México, D. F: McGraw-Hill.

Daboin, M., \& Romero, R. (2010). Estrategias gerenciales y motivación al logro en al logro en empleados de empresas manufactureras ubicadas en Maracaibo, Zulia (Tesis de maestría, Facultad de Ciencias Políticas, Administrativas y Sociales, Universidad Rafael Urdaneta. Maracaibo, Venezuela.). Recuperado de https://200.35.84.131/portal/php/buscar.php?base=marc\&cipar=marc.par\&epilo go $=\&$ Formato $=w \&$ Opcion $=$ detalle $\&$ Expresión $=! \mathrm{BDab}$ oin, + Mar\%Eda [Consulta: 16 de noviembre de 2020].

David, F.R. (2011).StrategicManagement: Conceptsand Cases. New Jersey: Prentice Hall. Recuperado de https://www. google.com.mx/url?sa=t\&source=web\&rct=j\&url=h ttp://irresearchers.ir/upload/docs/StrategicManagementDavid.pdf\&ved=0ahUKEwjl6JSt2_7XAhXhQd 8KHWgQB_UQFggnMAA\&usg=AOvVaw2lZjW_ 
Tq4WkBW8AxthZqDc [Consulta: 17 de noviembre de 2020].

Elizalde, H. (2011). Estrategia de crecimiento basada en el desarrollo de nuevos mercados para el PVC en México: segmento tintas (Tesis de maestría, Escuela Superior de Comercio y Administración, Instituto Politécnico Nacional. México, D. F., México). Recuperado de http://tesis.ipn.mx/xmlui/bitstream/handle/123456789/9327/XMESCA-ST-G\%20MAN\%20 E459h.pdf?sequence $=1$ \&isAllowed $=y$ [Consulta: 17 de noviembre de 2020].

Fernández, N. (2006). Politica, planeamiento y gestión de la educación. Caseros: UNTREF. Recuperado de https:// unesdoc.unesco.org/ark:/48223/pf0000150635 [Consulta: 16 de noviembre de 2020].

Fideicomiso de Riesgo Compartido (FIRCO). (8 de mayo de 2017). Agroindustria en México. Recuperado de https://www.gob.mx/firco/articulos/agroindustria-enmexico?idiom=es [Consulta: 16 de noviembre de 2020].

García, C. (2011). La medición en las ciencias sociales y en la psicología. En R. Landero \& M. González, Estadística con SPSS y metodología de la investigación (pp. 139166) México, D. F.: Trillas.

Hernández, R., Fernández, C., \& Baptista, P. (2014). Metodología de la investigación. México, D. F: McGraw-Hill.

Instituto Nacional de Estadística y Geografía (INEGI). (2014). Micro, pequeña, mediana y gran empresa. Estratificación de los establecimientos. Recuperado de www. inegi.org.mx [Consulta: 16 de noviembre de 2020].

Instituto Nacional de Estadística y Geografía (INEGI). (2016a). Directorio Estadístico Nacional de Unidades Económicas (DENUE). Recuperado de www.beta.inegi.org.mx [Consulta: 16 de noviembre de 2020].

Instituto Nacional de Estadística y Geografía (INEGI). (2016b). Esperanza de vida de los negocios en México. Recuperado de www.inegi.org.mx/inegi/contenidos/ investigación/Experimentales/Esperanza/default.aspx [Consulta: 16 de noviembre de 2020].
Lara, R. (2017, 24 de octubre). La tecnología es el "socio" que necesita el sector agroindustrial en México. Expansión. Recuperado de https:/expansion.mx/empresas/2017/ 10/24/la-tecnologia-es-el-socio-que-necesita-el-sectoragroindustrial-en-mexico?internal_source=PLAYLIST [Consulta: 17 de noviembre de 2020].

Marín, M. (2013, 21 de julio). Estrategias gerenciales. Estrategias Gerenciales: Cambio Organizacional. Recuperado de http://johannicolina.blogspot.mx/2013/07/estrategiasgerenciales.html [Consulta: 17 de noviembre de 2020].

Melo, L. I., \& Fonseca, D. E. (2014). Descripción y análisis de la gerencia en mipymes agroindustriales del departamento de Boyacá, Colombia. Acta Agronómica, 63 (4), 297-310. https://doi.org/10.15446/acag.v63n4.43382

Moral, J. (2011). Análisis factorial y su aplicación al desarrollo de escalas. En R. Landero \& M. González, Estadistica con SPSS y metodología de la investigación (pp. 387-443). México, D. F: Trillas.

Noguera, A. L., Barbosa, D. H., \& Castro, G. A. (2014). Estrategia organizacional: una propuesta de estudio. Estudios Gerenciales, 30 (131), 153-161. https://doi. org/10.1016/j.estger.2014.04.004

Organización de las Naciones Unidas para la Alimentación y la Agricultura (FAO). (2004). Calidad y competitividad de la agroindustria rural de América Latina y el Caribe: Uso eficiente y sostenible de la energía (Boletín de Servicios Agrícolas de la FAO 153). Recuperado de www.fao.org/docrep/008/y5603s/y5603s00.htm [Consulta: 16 de noviembre de 2020].

Prieto, J. E. (2011). Gestión estratégica organizacional. Bogotá: ECOE. Recuperado de https://www.ecoeedi ciones.com/wp-content/uploads/2017/05/Gesti\%C3\% B3n-estrat\%C3\%A9gica-organizacional-5ta-Edici \%C3\%B3n.pdf [Consulta: 18 de noviembre de 2020].

Riaño-Casallas, M. I., \& García-Ubaque, J. C. (2016). Gestión estratégica para hospitales universitarios. Revista de la Facultad de Medicina, 64 (4), 615-620. https:// doi.org/10.15446/revfacmed.v64n4.54816

Contabilidad y Negocios (16) 32, 2021 / ISSN 1992-1896 
Rubio, C. P. (2012). Gestión estratégica organizacional aplicada a las pymes constructoras en Colombia, (Tesis de maestría, Facultad Estudios en Ambientes Virtuales, Universidad de Nebrija. Madrid, España.). Recuperado de https://www.google.com.mx/url?sa=t\&sou rce=web \&rct=j\&url=http://repository.ean.edu.co/ bitstream/handle/10882/2845/RubioClaudia2012. pdf\%3Fsequence\%3D3\&ved=0ahUKEwjK3rq8M7XAhVL02MKHddBBG0QFggoMAA\&usg=AO vVaw2IBvdqvE7y0jaRAI0Ic1hN [Consulta: 22 de noviembre de 2020].

Sánchez, J. (2003). Estrategia integral para pymes innovadoras. Revista Escuela de Administración de Negocios, (47), 34-45. Recuperado de http://www.redalyc.org/articulo. oa?id=20604703 [Consulta: 22 de noviembre de 2020].

Serrano, I. O. (2017, 15 de noviembre). RSE en la red de valor de la agroindustria mexicana El Economista. Recuperado de https://www.eleconomista.com.mx/ opinion/RSE-en-la-red-de-valor-de-la-agroindustriamexicana-I-20171115-0116.html [Consulta: 22 de noviembre de 2020].

Suárez, S. (2019). Estrategias gerenciales de las micro y pequeñas empresas agroindustriales de Villahermosa, Tabasco (Tesis de maestría, División Académica de Ciencias Económico Administrativas, Universidad Juárez Autónoma de Tabasco. Villahermosa, México).
Recuperado de http://148.236.18.55/bitstream/20. 500.12107/3256/1/12_Tesis\%20Susana\%20Suarez. pdf [Consulta: 20 de noviembre de 2020].

Tarí, J. J., López, M. D., \& Molina, J. F. (2007). El proceso de autoevaluación según el modelo EFQM en una pyme. Investigaciones Europeas de Dirección y Economía de la Empresa, 13 (2), 203-216. Recuperado de https:// rua.ua.es./dspace/handle/10045/15477 [Consulta: 23 de noviembre de 2020].

Thompson, A. A., Peteraf, M. A., Gamble, J. E. \& Strickland, A. J. (2015). Administración estratégica. Teoría y casos. México, D. F.: McGraw-Hill

Urdaneta, A. (2013). Estrategias gerenciales basadas en la calidad de gestión de la pequeña y mediana empresa de Acarigua (Tesis de maestría, Universidad Nacional Experimental de los Llanos Occidentales Ezequiel Zamora. Barinas, Venezuela). Recuperado de http://app.vpa.unellez.edu. ve/bibliotecavpa/opac_css/doc_num.php?explnum_ $\mathrm{id}=52$ [Consulta: 21 de noviembre de 2020].

Fecha de recepción: 02 de marzo de 2021 Fecha de aceptación: 08 de agosto de 2021 Correspondencia: susy.93suarez@gmail.com maria.sandoval@ujat.mx cluzlamoyi@gmail.com 